\title{
Long-term fluorescence of erythrosine in alive mammalian cells
}

\author{
Valeriya Maryakhina \\ Orenburg State University, 13 Pobedy st., Orenburg, 460018, Russia \\ e-mail: valemar@mail.ru
}

\begin{abstract}
Investigation of triplet states of probes molecules located in cells and tissues is used for research of pathological processes included cancer development due to high sensitivity of triplet states lifetime to surround medium. In the work the long-term fluorescence of erythrosine located in alive mammalian cells has been analyzed by mathematical simulation. The model considers all basic processes of relaxation of probe triplet states in biological media. It is obtained that signal intensity and its form depends on oxygen concentration and viscosity of cells cytoplasm in which the dye diffuses. Interaction of erythrosine with the variety of extinguishers (included immobilization process into peptides molecules) does not impact on signal of the delayed fluorescence. Their concentration impact on cell viscosity mean only. The most important extinguisher is molecular oxygen impacting on signal. The correlation between theoretical and experimental results let to consider that the model is adequate. (C) 2016 Journal of Biomedical Photonics \& Engineering.
\end{abstract}

Keywords: long-term fluorescence, erythrosine, singlet oxygen, mammalian cells, fluorescent diagnostics.

Paper \#3078 received 2016.07.18 revised manuscript received 2016.09.22; accepted for publication 2016.09.29; published online 2016.09.30. doi: 10.18287/JBPE16.02.030303

\section{References}

1. M. Scholz, A.-L. Biehl, R. Dědic, and J. Hála, "The singlet-oxygen-sensitized delayed fluorescence in mammalian cells: a time-resolved microscopy approach,” Photochem. Photobiol. Sci. 14(4), 700-713 (2015).

2. F. A. Harms, W. M. de Boon, G. M. Balestra, S. I. Bodmer, T. Johannes, R. J. Stolker, and E. G. Mik, "Oxygen-dependent delayed fluorescence measured in skin after topical application of 5-aminolevulinic acid," J. Biophotonics 4(10), 731-739 (2011).

3. V. V. Bryukhanov, G. A. Ketsle, V. C. Laurinas, L. V. Levshin, and Z. M. Muldakhmetov, "Singlet-triplet annihilation of singlet oxygen and triplet xanthene dye molecules in liquid solutions," Journal of Applied Spectroscopy 46(4), 372-377 (1987).

4. E. A. Ermilov, O. L. Markovskii, and I. M. Gulis, "Inductive-resonant triplet-triplet annihilation in solid solutions of erythrosine," Journal of applied spectroscopy 64(5), 642-645 (1997).

5. F. Kazmi, L. J. Haupt, J. R. Horkman, B. D. Smith, D. B. Buckley, E. A. Wachter, and J. M. Singer,. "In vitro inhibition of human liver cytochrome P450 (CYP) and UDP-glucuronosyltransferase (UGT) enzymes by rose bengal: system-dependent effects on inhibitory potential," Xenobiotica 44(7), 606-614 (2014).

6. J. Lee, I. Kwon, S. S. Jang, and A. E. Cho, "Investigation of the effect of erythrosine B on amyloid beta peptide using molecular modeling," Journal of molecular modeling 22(4), 1-9 (2016).

7. S. N. Letuta, and V. S. Maryakhina, "The delayed fluorescence kinetics as a method of biological tissue diagnostics", Proc. SPIE 7999, 799907 (2011).

8. S. N. Letuta, V. S. Maryakhina, and R. R. Rakhmatullin, "Optical diagnostics of biological tissue cells during their cultivation in polymers," Quantum electronics 41(4), 314-317 (2011).

9. K. Rohatgi Mukherjee, and A. K. Mukhopadhyay, "Photophysical processes in halofluorescein dyes," Indian Journal of Pure \& Applied Physics 14(6), 481-484 (1976).

10. I. M. Vlasova, and A. M. Saletsky, "Fluorescent characteristics of probes of the fluorescein family in human serum albumin solutions,” Moscow Univ. Phys. Bull. 64, 412-416 (2009). 
11. V. V. Bryukhanov, G. A. Ketsle, V. C. Laurinas, and L. V. Levshin, "Oxygen induction of delayed fluorescence of fluorescenic dye liquid solutions," Optika i Spektroskopiya 60(1), 205-207 (1986).

12. A. M. Mastro, M. A. Babich, W. D. Taylor, and A. D. Keith, "Diffusion of a small molecule in the cytoplasm of mammalian cells," Proc. Natl. Acad. Sci. 81(11), 3414-3418 (1984).

13. A. G. Melnikov, A. B. Pravdin, V. I. Kochubey, A. V. Kuptsova, and G. V. Melnikov, "Interglobular diffusion of an energy donor in triplet-triplet energy transfer in proteins," J. Spectrosc. 2013, 1-7 (2013).

14. A. A. Krasnovsky, "Luminescence and photochemical studies of singlet oxygen photonics," Journal of Photochemistry and Photobiology A: Chemistry 196(2-3), 210-218 (2008).

15. S. Suresh, "Biomechanics and biophysics of cancer cells," Acta Biomaterialia 3(4), 413-438 (2007).

16. R. B. Gorenkov, V. N. Karpov, D. A. Rogatkin, and V. I. Shumskiy, "Chronic hypoxia state as one of the factors of increased fluorescence of endogenous porphyrins in alive biological tissues," Biophysics 52(4), 711714 (2007).

\section{Introduction}

Investigation of triplet states of probes molecules located in cells and tissues is used for research of pathological processes included cancer development [see, for example, 1, 2]. It can be possible due to high sensitivity of triplet states lifetime to surround medium. Nowadays, it is considered that lifetime of triplet states of fluorescein derivatives (erythrosine, eosine and rose bengal) located in cells depends on oxygen and peptide concentration in medium primarily because it is a basic quenchers of triplet states. So, mean of erythrosine lifetime may be from $4 \mu$ s in oxygen containing medium [3] to 700 [4] $\mu \mathrm{s}$ in polymethylmethacrylate where oxygen concentration is a little. Interaction of fluoresceine derivative with peptide had also investigated in detail $[5,6]$. However, change of lifetime of probe triplet states can be as either positive or negative role in biomedical diagnostics. On one hand, it can be as an indicator of change of cell cytoplasm for pathology present. On another hand, data of spectralkinetic measurements are different even at a little change of parameter, for example, room temperature. It creates additional error in signal registration. Because of it, the most important limited processes with fluorophores triplet states participation at registration of long-term fluorescence need to know. This task can be solved by different experimental methods but it requests huge quantities of additional experiments.

Thus, the aim of the research is definition of the basic photophysical processes at registration of longterm fluorescence of erythrosine impacting on signal formation by the mathematical simulation. These results were obtained in according with our preview experimental results [7] for development of the optical techniques of biomedical diagnostics of pathologies.

\section{The object and methods of experimental investigation}

Normal and cancer mammalian cells were obtained by enzyme treatment of respective tissues cells of female mice (line BYRB) with spontaneous malignant tumors during 40 minutes at $37^{\circ} \mathrm{C}$. After that the received cell suspension was placed on the nutrient medium (biomaterial "Hyamatrix" [8]) and was incubated during several days at $37{ }^{\circ} \mathrm{C}$. The fragmants of biomaterial with cultivated cells were coloured by erythrosine water solution with the concentration $10^{-4} \mathrm{M}$. This dye was selected due to its high quantum yield in triplet state, intensive phosphorescence, high dissolving degree in water and penetration possibility into cells [9].

Registration of kinetics of the delayed fluorescence of dye was after radiation by the second harmonic of Nd: YAG laser $(\lambda=532 \mathrm{~nm})$. Wave length of registration was $560 \mathrm{~nm}$. Laser impulse was with durability $10 \mathrm{~ns}$ and energy $10 \mathrm{mDg}$. The samples for study were placed in a special evacuated chamber. The air pressure (oxygen concentration) above the surface of coloured biopolymer changed from 0.3 to $1.0 \mathrm{~atm}$. Description of the device in detail is in [8].

\section{The experimental results}

The experimental curves of long-term fluorescence of erythrosine located in cancer and normal mammary cells are depicted on figure 1. As you can see, the curves have hump-shaped type. It means that processes with molecular oxygen participation are during signal registration. For coloring cells, the part of nutrient medium can be also colored. Because of it, additionally the long-term fluorescence was measured from nutrient medium colored by erythrosine. As we described earlier [8], the kinetics has hyperbolic type and does not depend on pressure above samples. Consequently, it defines by annihilation of near-located of triplet molecules of dye only. Nature of the long-term fluorescence of erythrosine located in cells is more interest and does not define unambiguously. In the next section analysis of the fluorescence signal is described by numerical simulation.

\section{The mathematical model}

Erythrosine molecule is excited to the first singlet state $\mathrm{S} 1$ and transfers to the first triplet state $\mathrm{T} 1$ by way $\mathrm{S} 0 \rightarrow$ $\mathrm{S} 1 \rightarrow \mathrm{T} 1$ (Fig. 2) after laser impulse. Since lifetime of erythrosine is more in three times than laser impulse duration, we will describe processes being after laser impulse, that is, processes of relaxation of triplet states will be considered only. 


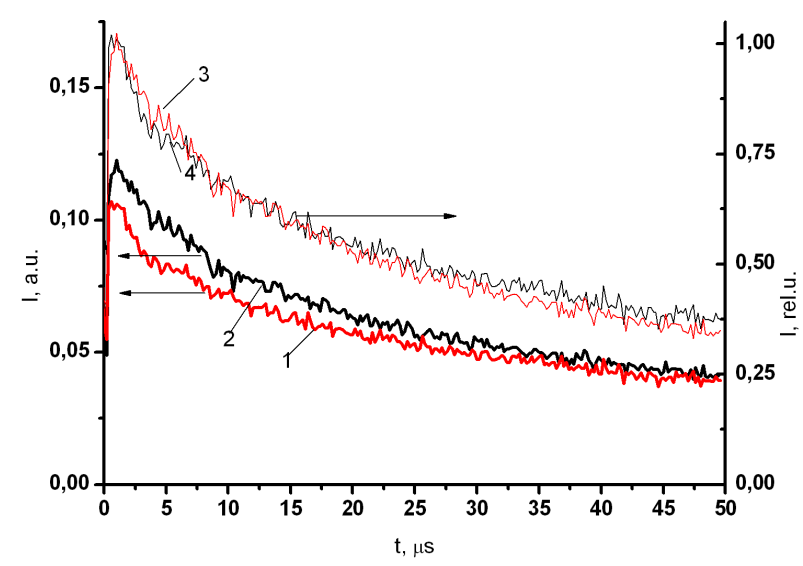

Fig. 1 The experimental curves of long-term fluorescence kinetics of erythrosine located in normal mammalian cells under air pressure above samples 0.5 atm (1) и 0.8 atm (2). The curves (3) and (4) are normalized curves (1) and (2).

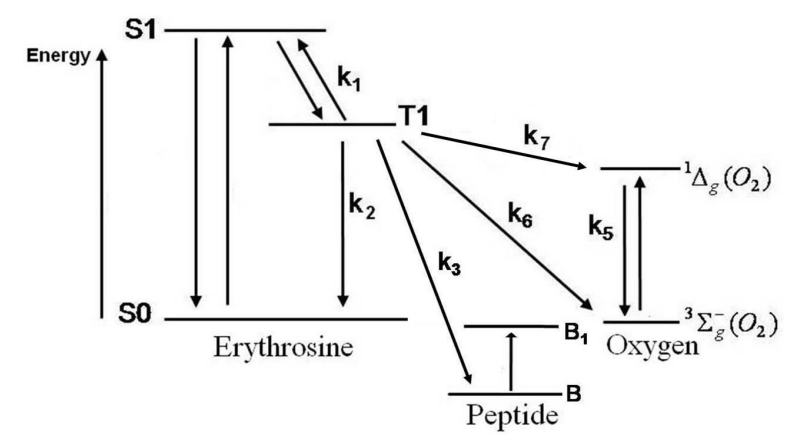

Fig. 2 Energy levels of erythrosine with possible energy transfers to quenchers such as peptide and oxygen.

The long-term fluorescence is sum signal of emission of probes with triplet states participation. Now all these processes will be described. So, xanthene dyes have a singlet-triplet splitting (energy difference between singlet and triplet state) $\sim 3000 \mathrm{~cm}^{-1}$ with exhibit thermoactivated delayed fluorescence [9]

$$
\mathrm{T} 1 \stackrel{\mathrm{k}_{1}}{\longrightarrow} \mathrm{S} 1 \longrightarrow \mathrm{S} 0
$$

and phosphorescence

$$
\mathrm{T} 1 \stackrel{\mathrm{k}_{2}}{\longrightarrow} \mathrm{S} 0 \text {. }
$$

These processes are monomolecular with lifetime depended on quenchers presence.

In the model the fast fluorescence with energy transfer $\mathrm{S} 1 \rightarrow \mathrm{S} 0$ will not be considered because it is during few nanoseconds in front of the described processes (1-2) being in microsecond region.

There are many different quenchers in cell cytoplasm with which probes molecules can interact. After this interaction probes molecules become unavailable for oxygen. These quenchers for the probe can be peptides (B) [10]. Hence, energy of the excited state of erythrosine transfers to peptide molecule with transformation to molecule $\mathrm{B} 1$. This process can be depicted as

$$
\mathrm{T} 1+\mathrm{B} \stackrel{\mathrm{k}_{3}}{\longrightarrow} \mathrm{S} 0+\mathrm{B} 1 .
$$

The next possible process is triplet-triplet annihilation of migrated molecules of dyes with last generation of their basic and excited singlet states for reaction

$$
\mathrm{T} 1+\mathrm{T} 1 \stackrel{\mathrm{k}_{4}}{\longrightarrow} \mathrm{S} 1+\mathrm{S} 0 .
$$

The generated S1 states impact on kinetics of longterm fluorescence [4] for reaction (1).

Oxygen is a very efficient quencher of triplet states of fluorophores. Their quenching is accompanied by a photosensitized generation of singlet oxygen. As a result of reaction between triplet states of molecules and molecular oxygen

$$
\mathrm{T} 1+{ }^{3} \mathrm{O}_{2} \stackrel{\mathrm{k}_{6}}{\longrightarrow} \mathrm{S} 0+{ }^{1} \Delta_{\mathrm{g}}
$$

singlet oxygen ${ }^{1} \Delta_{g}\left(O_{2}\right)$ is generated.

Next, the non-extinguished triplet states of molecules can interact either with migrated ${ }^{1} \Delta_{g}\left(O_{2}\right)$ singlet oxygen (reaction of singlet-triplet annihilation)

$$
{ }^{1} \Delta_{\mathrm{g}}+\mathrm{T} 1 \stackrel{\mathrm{k}_{7}}{\longrightarrow} \mathrm{S} 1+{ }^{3} \mathrm{O}_{2},
$$

additionally, influence on the delayed fluorescence. As the result of annihilation of migrating singlet oxygen with triplet-excited dye molecules, singlet $S_{1}$ states of fluorophores can be formed, which make an additional contribution to the delayed fluorescence [11]. Finally, the kinetic curve is transformed acquiring a hump shape.

The generated singlet oxygen also relaxes to basic state

$$
{ }^{1} \Delta_{\mathrm{g}} \stackrel{\mathrm{k}_{5}}{\longrightarrow}{ }^{3} \mathrm{O}_{2} \text {. }
$$

Kinetics of the delayed fluorescence of the triplet probe located in normal and cancer mammalian cells are different [7] because of oxygen concentration changing within it. On the basis of the described processes, system of balance kinetic equations can be written as 


$$
\begin{aligned}
& \frac{d N_{T}}{d t}=-\left(k_{6} N_{O_{2}}+k_{7} N_{\Delta}\right) N_{T}-2 k_{4} N_{T}^{2}-k_{3} N_{T} N_{B}-k_{2} N_{T} \\
& \frac{d N_{O_{2}}}{d t}=-k_{6} N_{T} N_{O_{2}}+\left(k_{7} N_{T}+k_{5}\right) N_{\Delta} \\
& \frac{d N_{\Delta}}{d t}=k_{6} N_{T} N_{O_{2}}-\left(k_{7} N_{T}+k_{5}\right) N_{\Delta} ; \\
& \frac{d N_{0}}{d t}=k_{6} N_{T} N_{O_{2}}+k_{4} N_{T}^{2}+k_{3} N_{T} N_{B}+k_{2} N_{T} \\
& \frac{d N_{1}}{d t}=k_{7} N_{\Delta} N_{T}+k_{4} N_{T}^{2} \\
& \frac{d N_{B}}{d t}=-k_{3} N_{T} N_{B} \\
& \frac{d N_{B_{1}}}{d t}=k_{3} N_{T} N_{B}
\end{aligned}
$$

where $k_{5}=1 / \tau_{\Delta}-$ singlet oxygen relaxation $\left(s^{-1}\right), k_{7}-$ rate constant of singlet-triplet annihilation $\left(\mathrm{cm}^{3} \mathrm{~s}^{-1}\right), k_{6^{-}}$ rate constant of quenching triplet states of erythrosine by molecular oxygen $\left(\mathrm{cm}^{3} \mathrm{~s}^{-1}\right), N_{T}, N_{0}, N_{1}$, - quantity of molecules in triplet, basic and the first excited state of dye $\left(\mathrm{cm}^{-3}\right), N_{B}, N_{B 1}$ - quantity of molecules in basic and excited states of peptides $\left(\mathrm{cm}^{-3}\right) ; k_{2}$ - rate constant of phosphorescence $\left(s^{-1}\right), k_{4}$ - rate constant of triplet-triplet annihilation $\left(s^{-1}\right), k_{3^{-}}$rate constant of energy transfer from erythrosine to peptide $\left(\mathrm{cm}^{3} \mathrm{~s}^{-1}\right)$.

For solution of the system of differential equations means of the parameters need to know. Now we will describe theirs in detail. First of all, phosphorescence is monomolecular process with inversely proportional rate to the lifetime of triplet states of molecules. The lifetime is $700 \mu \mathrm{s}$ for quenchers absent [4]. Thus,

$$
k_{2}=\frac{1}{\tau_{p h}}=\frac{1}{700 \cdot 10^{-6}}=0.14 \cdot 10^{4} s^{-1} .
$$

The rate constant of triple-triplet annihilation can be calculated by formulae

$$
k_{4}=\frac{2 k T}{3 \eta} \cdot \frac{r+r_{2}}{r \cdot r_{2}}=3.9 \cdot 10^{-12} \mathrm{~cm}^{3} \mathrm{~s}^{-1}
$$

where $r_{i}$ - radiuses of molecules interacting each other, $\mathrm{nm}$ (for this case $r=r_{2}$ ); $k$ - Boltzmann constant; $T$ temperature, $\mathrm{K} ; \eta$ - viscosity, Pz. We assume that temperature was $330 \mathrm{~K}$. Viscosity of cell cytoplasm was from 1 to $8^{\circ} \mathrm{cP}$ in according to work [12]. Radius of erythrosine molecule was calculated for its density $\rho$ in dry state and molecular weight $\mathrm{M}$ as

$$
r=\sqrt[3]{\frac{3 \cdot M}{4 \cdot \rho \cdot \pi \cdot N_{A}}} .
$$

The rate coefficient $k_{3}$ was obtained by Melnikov A.G. with coauthors and is $3 \cdot 10^{6} \mathrm{M}^{-1} \mathrm{~s}^{-1}[13]$.
In point of investigation results of Krasnovsky we can conclude that lifetime of singlet oxygen in somatic cells is nearly $3 \mu \mathrm{s}$ [14]. Thus, rate of singlet oxygen relaxation is

$$
k_{5}=\frac{1}{\tau_{\Delta}}=\frac{1}{3 \cdot 10^{-6}}=0.3 \cdot 10^{6} s^{-1}
$$

The rate constants $k_{6}, k_{7}$ can be also calculated for kinetic method as well as $k_{4}$. Because of equality of molecule radiuses of singlet and triplet oxygen, these coefficients will be equal and are

$$
k_{6}=k_{7}=16.0 \cdot 10^{-21} \mathrm{~cm}^{3} \mathrm{~s}^{-1}
$$

We note that coefficients $k_{3}, k_{5}$ and $k_{2}$ are significantly higher than other coefficients $k_{4}, k_{6}$ and $k_{7}$. Consequently, they cannot be considered in the researched time region. Thus, the system of equations (7) can be simplify and is

$$
\begin{aligned}
& \frac{d N_{T}}{d t}=-\left(k_{6} N_{O_{2}}+k_{7} N_{\Delta}\right) N_{T}-2 k_{4} N_{T}^{2} ; \\
& \frac{d N_{O_{2}}}{d t}=-k_{6} N_{T} N_{O_{2}}+k_{7} N_{T} N_{\Delta} ; \\
& \frac{d N_{\Delta}}{d t}=k_{6} N_{T} N_{O_{2}}-k_{7} N_{T} N_{\Delta} ; \\
& \frac{d N_{0}}{d t}=k_{6} N_{T} N_{O_{2}}+k_{4} N_{T}^{2} ; \\
& \frac{d N_{1}}{d t}=k_{7} N_{\Delta} N_{T}+k_{4} N_{T}^{2}
\end{aligned}
$$

with initial conditions

$$
\begin{aligned}
& N_{T}(0)=1 ; \\
& N_{1}(0)=0 ; \\
& N_{0}(0)=0 ; \\
& N_{\Delta}(0)=0 ; \\
& N_{O_{2}}(0)=X .
\end{aligned}
$$

Since accurate initial concentration of oxygen in somatic cells is unknown, the value is marked as $\mathrm{X}$. In the model we can consider ratio between $\mathrm{N}_{\mathrm{T}}$ and $\mathrm{N}_{\mathrm{O} 2}$ only. All calculations will be carried out in according to the ratio.

Finally, kinetics of long-term fluorescence of molecules is given as

$$
I_{D F}(t)=\varphi_{f l} k_{5} N_{T}(t) N_{\Delta}(t)+k_{4} N_{T}^{2}(t)+\varphi_{f l} k_{1} N_{T}(t)
$$

where $\varphi_{f l}$ - quantum yield of fluorescence.

The first summand in (9) is annihilation contribution with singlet oxygen participation; the second summand is triplet-triplet annihilation and the third- 
termoactivation process. Since in somatic cells intercombinational conversion is significantly faster than diffusion processes, final signal will be a sum of the first and the second summand.

\section{Numerical results and their discussion}

In the figure 3 the calculated curves of long-term fluorescence of erythrosine for viscosity mean $2 \mathrm{cP}$ are shown for different oxygen concentrations. Signal intensity increases when oxygen concentration rises. It is concluded that oxygen concentration impacts on signal intensity primarily. These curves are identical after their normalizing (curve 4). This result is unexpected and contradics to work [3] but the experimental curves have similar dependence (fig. 1) in which oxygen pressure above samples is 0.5 and 0.8 atm. Time of relaxation of triplet states is significantly different because these curves were calculated for means of viscosity and ratio $\mathrm{N}_{\mathrm{t}}: \mathrm{N}_{0}$ differ from these means for cells. The next analysis will show accurate parameters means.

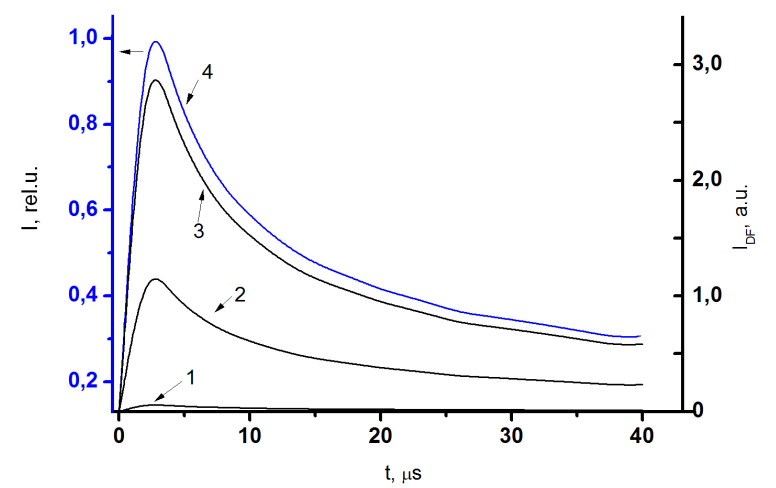

Fig. 3 The calculated curves of long-term fluorescence of erythrosine for viscosity mean $2 \mathrm{cP}$ for ratio: $1-\mathrm{N}_{\mathrm{t}}: \mathrm{N}_{0}$ $=1: 0.001 ; 2-\mathrm{N}_{\mathrm{t}}: \mathrm{N}_{0}=1: 0.005 ; 3-\mathrm{N}_{\mathrm{t}}: \mathrm{N}_{0}=1: 0.01$. Curve (4) is normalized curves (1-3). The curves are connected by B-spline.

Earlier Bryukhanov with coauthors [3] had shown that maximum of long-term fluorescence rises and shifts to short time region in polymer media when oxygen concentration increases. However, biological samples differ for viscosity not only but metabolic processes are also. As a result, oxygen concentration within it is constantly changing. It is reason to assume that oxygen concentration is not unique factor impacting on form of the delayed fluorescence signal.

The most sensitive parameter to the delayed fluorescence is cytoplasm viscosity. Signal intensity is changed not only but its form is changed also (Fig. 4). All curves on the figure are connected by B-spline function. Dots are not depicted in some curves for most visibility.

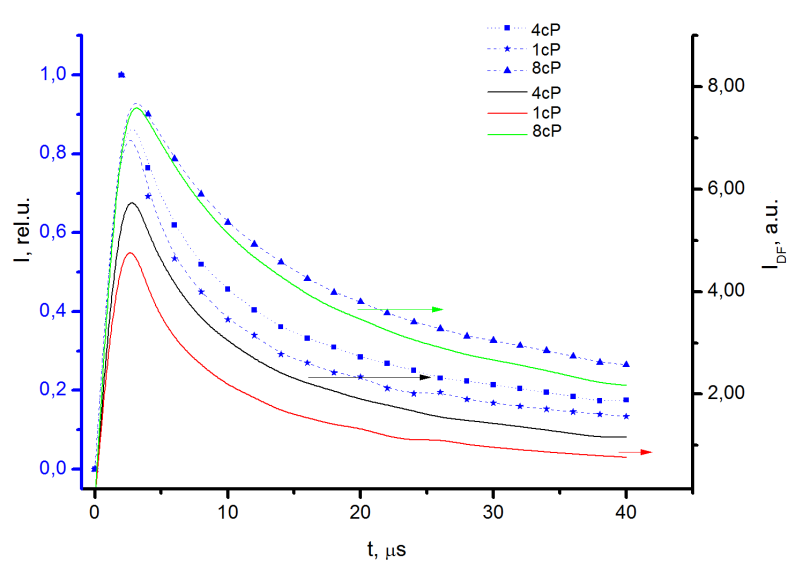

Fig. 4 The calculated curves of erythrosine located in somatic cells for viscosity mean $1,2,4 \mathrm{cP}$ and ratio oxygen concentration $\mathrm{N}_{\mathrm{T}}: \mathrm{N}_{\mathrm{O} 2}=1: 0.001$. Solid lines are the calculated curves; dotted lines are the curves after their normalizing.

Viscosity is parameter from which diffusion coefficient depends. Viscosity decreasing lead to delay diffuse-controlled processes such us triplet-triplet and singlet-triplet annihilations (eq. 3-5). As a result, decline of kinetics from 5 to $40 \mu$ s is a gently sloping but curve maximum is slower and shifts to long-time region.

In the figure 5 the experimental and calculated curves of erythrosine located in normal and cancer mammalian cells have shown. As you can see, the calculated curves as well as the experimental kinetics are crossed at $10 \div 12 \mu \mathrm{s}$. The most positive correlation is for normal cells $\mathrm{N}_{\mathrm{T}}: \mathrm{N}_{\mathrm{O} 2}=1: 0.02$ and $2 \mathrm{cP}$; for cancer cells $\mathrm{N}_{\mathrm{T}}: \mathrm{N}_{\mathrm{O} 2}=1: 0.001$ and $6 \mathrm{cP}$. In according to this calculation, cytoplasm viscosity of cancer cells is significantly more than for normal cells. It is confirmed by data of another scientific group [15]. In another work data about viscosity of cancer cells are as $4.5 \mathrm{cP}$ [12]. Furthermore, $\mathrm{N}_{\mathrm{T}}: \mathrm{N}_{\mathrm{O} 2}$ for cancer cells is less than for normal cells in 5 times, i.e., oxygen concentration decreasing is looked at. Really, cancer cells differ from normal cell by hypoxia [16] connected on accelerated oxygen consumption and its concentration decreasing.

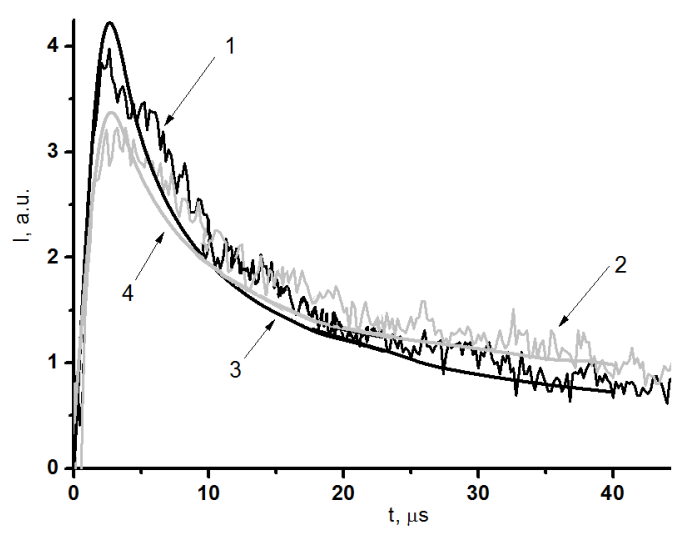

Fig. 5 The experimental $(1,2)$ and calculated $(3,4)$ curves of long-term fluorescence of eruthrosine in normal $(1,3)$ and cancer $(2,4)$ mammalian cells measured for atmosphere pressure above samples. 


\section{Conclusion}

In the work we descussed all basic processes which can impact on to form signal of the delayed fluorescence of xanthene dyes. Inspite of variety of quenchers in cells, extinguishing by molecular oxygen lead to signal changing only. Interaction of erythrosine with other quenchers, included peptides, unsignificant impacts on the signal. On another hand, peptides and organells concentration increasing lead to viscosity rising. This factor also impacts on diffusion-controlled processes and signal forming.

The obtained results can be useful for investigation of fluorescence signals of triplet probes and photosensitizers in biological media and for development of optical techniques of biomedical diagnostics.

\section{Acknowledgments}

Author thanks prof. Letuta S.N. for discussing results. The work is partically supported by President fellowship SP-2015-273.4 and Ministry of education and science of Russia. (agreement №450). 